\title{
An attitude determination method based on convected Euler angle error model for SINS/CNS integrated system
}

\author{
Jianli Li ${ }^{1}$, Yun Wang ${ }^{1}$, Pengfei Dang ${ }^{1}$, Zhaoxing Lu ${ }^{1,2}$ \\ 1 School of Instrument Science and Opto-electronic Engineering, Beijing University of Aeronautics and Astronautics, Beijing 100191, China \\ 2 Xi'an Institute of Hi-tech, Xi'an 710025, China \\ *Correspondence: lijianli@buaa.edu.cn; Tel.: +86-10-82339550
}

\begin{abstract}
The attitude determination method plays an important role in SINS/CNS integrated system for spacecraft. Since the misalignment angels are indirect measurements, the misalignment angle model used in the existing attitude determination method can cause transformation errors. To solve the problem, an attitude determination method based on convected Euler angle error model for SINS/CNS integrated system is proposed. The attitude error propagation is analyzed, and the convected Euler angle error model is derived. Furthermore, the state equation of SINS/CNS integrated system is established. The Kalman filter estimates and compensates the Euler angle errors. Finally, simulation results verified that the proposed method can improve the attitude accuracy compared to the conventional misalignment angle method.
\end{abstract}

Keywords: Attitude determination method; SINS/CNS integrated system; Misalignment angels; Transformation errors; Convected Euler angle errors

\section{Introduction}

The strapdown inertial navigation system (SINS) can continuously provide comprehensive navigation parameters using gyros and accelerometers, and is an important self-contained navigation technology, but its navigation error grows with the time ${ }^{[1,2]}$. The celestial navigation system (CNS) is a conventional autonomous navigation method, and it can provide high precision attitude, but strongly depends on the measurement accuracy of sensors ${ }^{[3,4]}$. In order to achieve a high precision attitude determination capability, SINS/CNS integrated method is used for the spacecraft ${ }^{[5]}$. CNS utilizes direct measurement of celestial using star sensor to obtain high-accuracy attitude autonomously, and realizes comprehensive correction of SINS attitude ${ }^{[6]}$. Meanwhile, SINS provides high frequency attitude information to make up the low data rate of $\mathrm{CNS}^{[7]}$.

The attitude error model plays an important role in implementing the Kalman filter for a SINS/CNS integrated system $^{[8-10]}$. However, most of the existing attitude error models focus on misalignment angles which cannot directly describe the attitude error propagation ${ }^{[11]}$. Since the attitude matrix is deduced by the misalignment angles, the attitude matrix of SINS cannot be seen as the mathematic reference directly ${ }^{[7]}$. Combining the CNS with SINS, the Kalman filter estimates the errors of misalignment angles in order to compensate the attitude matrix, but the process of attitude propagation is not described intuitively. Then, Wang proposed a SINS/CNS integration model based on misalignment angle errors, which avoids attitude transformation, but errors contained in star observations will be transferred into a determined attitude matrix ${ }^{[12]}$. From the above it can be obtained that the misalignment angle method is not intuitive enough to analyze attitude propagation. The key issue is transformation errors between Euler angle errors and misalignment

\footnotetext{
Copyright (C) 2018 Jianli Li et al.

doi: $10.18063 /$ som.v3i2.694

This is an open-access article distributed under the terms of the Creative Commons Attribution Unported License

(http://creativecommons.org/licenses/by-nc/4.0/), which permits unrestricted use, distribution, and reproduction in any medium, provided the original work is properly cited.
} 
angles, when the high-order attitude error compensation is necessary for high-accuracy navigation ${ }^{[13,14]}$. The Euler angle errors can directly reflect attitude determination processing ${ }^{15,16]}$. Therefore, the Euler angles are intuitive while analyzing the attitude propagation in SINS/CNS integrated system, just as the satellite is represented by Euler angles in the space-based environment ${ }^{[17]}$. The Euler angles as direct measurement of attitude can reflect the attitude transfer law, and the analysis of Euler angle errors is necessary in SINS/CNS integrated system.

To solve the problem, an attitude determination method based on convected Euler angle error model for SINS/CNS integrated system is proposed. Firstly, in view of the operation principles of SINS in inertial frame (i-frame), a differential formulation of convected Euler angle errors is proposed. Then, the Kalman filter is designed and implemented for SINS/CNS integrated system based on the convected Euler angle error differential equation, where both state and measurement are Euler angle errors. Finally, the simulation experiment is carried out to verify the proposed method, and the results show that the proposed method is superior to the conventional misalignment angle method.

\section{The convected Euler angle error modeling}

In order to analyze the attitude error propagation, it is necessary to derive the Euler angle error differential equations. The body attitude matrix $C_{i}^{b}$ used in SINS/CNS integrated system represents an attitude relation of the body frame (b-frame) respect to i-frame. As show in Fig.1, the body attitude matrix $C_{i}^{b}$ from i-frame to b-frame can be

$$
C_{i}^{b}=R_{x}^{2}(\gamma) R_{y}^{1}(\varphi) R_{z}^{i}(\theta)=\left[\begin{array}{ccc}
\text { calculated by Euler angles } \varphi, \theta & \text { and } \gamma & \text { along head, pitch and roll: } \\
\cos (\varphi) \cos (\theta) & \cos (\varphi) \sin (\theta) & -\sin (\varphi) \\
\sin (\gamma) \sin (\varphi) \cos (\theta)-\cos (\gamma) \sin (\theta) & \sin (\gamma) \sin (\varphi) \sin (\theta)+\cos (\gamma) \cos (\theta) & \sin (\gamma) \cos (\varphi) \\
\cos (\gamma) \sin (\varphi) \cos (\theta)+\sin (\gamma) \sin (\theta) & \cos (\gamma) \sin (\varphi) \sin (\theta)-\sin (\gamma) \cos (\theta) & \cos (\gamma) \cos (\varphi)
\end{array}\right]
$$

where the i-frame has its origin at launch point, its $\mathrm{x}$-axis aims towards the expected target, $\mathrm{y}$-axis is a vertical by launch point, and z-axis complements the right-handed system. The b-frame has its origin at the center of body, its $x$-axis points to head along longitudinal axis, y-axis is perpendicular to the longitudinal plane of symmetry and z-axis complements the right-handed system ${ }^{[5]} . R_{x}^{2}(\gamma), R_{y}^{1}(\varphi)$ and $R_{z}^{i}(\theta)$ respectively represent attitude matrix induced by rotation Euler angles $\gamma, \varphi$ and $\theta$ along roll, head and pitch.

In order to accurately derive the Euler angle error differential equations, the transformation relation between the angular velocity of b-frame respect to i-frame denoted in b-frame and the Euler angle velocities in head, pitch and roll with respect to i-frame in initial position, can be expressed as:

$$
\left[\begin{array}{c}
\omega_{i b x}^{b} \\
\omega_{i b y}^{b} \\
\omega_{i b z}^{b}
\end{array}\right]_{b}=R_{x}^{2}(\gamma) R_{y}^{1}(\varphi) R_{z}^{i}(\theta)\left[\begin{array}{c}
0 \\
0 \\
\dot{\theta}
\end{array}\right]_{i}+R_{x}^{2}(\gamma) R_{y}^{1}(\varphi)\left[\begin{array}{c}
0 \\
\dot{\varphi} \\
0
\end{array}\right]_{1}+R_{x}^{2}(\gamma)\left[\begin{array}{c}
\gamma^{\cdot} \\
0 \\
0
\end{array}\right]_{2}
$$

where $\omega_{i b j}^{b}$ is angular velocity of $\mathrm{b}$-frame respect to $\mathrm{i}$-frame denoted in $\mathrm{b}$-frame along $\mathrm{j}$ axis $(\mathrm{j}=\mathrm{x}, \mathrm{y}, \mathrm{z})$, and can be directly measured by gyros. The equation (2) can be rewritten by Euler angles:

$$
\left[\begin{array}{c}
\omega_{i b x}^{b} \\
\omega_{i b y}^{b} \\
\omega_{i b z}^{b}
\end{array}\right]_{b}^{b}=\left[\begin{array}{ccc}
1 & 0 & 0 \\
0 & \cos (\gamma) & \sin (\gamma) \\
0 & -\sin (\gamma) & \cos (\gamma)
\end{array}\right]\left[\begin{array}{ccc}
\cos (\varphi) & 0 & -\sin (\varphi) \\
0 & 1 & 0 \\
\sin (\varphi) & 0 & \cos (\varphi)
\end{array}\right]\left[\begin{array}{l}
0 \\
0 \\
\dot{\theta}
\end{array}\right]_{i}+\left[\begin{array}{ccc}
1 & 0 & 0 \\
0 & \cos (\gamma) & \sin (\gamma) \\
0 & -\sin (\gamma) & \cos (\gamma)
\end{array}\right]\left[\begin{array}{c}
0 \\
\dot{\varphi} \\
0
\end{array}\right]_{1}+\left[\begin{array}{c}
\dot{\gamma} \\
0 \\
0
\end{array}\right]_{2}=\left[\begin{array}{ccc}
0 & -\sin (\varphi) & 1 \\
\cos (\gamma) & \sin (\gamma) \cos (\varphi) & 0 \\
-\sin (\gamma) & \cos (\gamma) \cos (\varphi) & 0
\end{array}\right]\left[\begin{array}{c}
\dot{\varphi} \\
\dot{\theta} \\
\dot{\gamma}
\end{array}\right]
$$

According to equation (3), the Euler angle velocities induced by angular velocities of $b$-frame respect to $\mathrm{i}$-frame denoted in b-frame can be written as:

$$
\left[\begin{array}{c}
\dot{\varphi} \\
\dot{\theta} \\
\dot{\gamma}
\end{array}\right]=\left[\begin{array}{ccc}
0 & -\sin (\varphi) & 1 \\
\cos (\gamma) & \sin (\gamma) \cos (\varphi) & 0 \\
-\sin (\gamma) & \cos (\gamma) \cos (\varphi) & 0
\end{array}\right]^{-1}\left[\begin{array}{c}
\omega_{b b x}^{b} \\
\omega_{i b y}^{b} \\
\omega_{i b z}^{b}
\end{array}\right]_{b}^{b}=\frac{1}{\cos (\varphi)}\left[\begin{array}{ccc}
0 & \cos (\varphi) \cos (\gamma) & -\cos (\varphi) \sin (\gamma) \\
0 & \sin (\gamma) & \cos (\gamma) \\
\cos (\varphi) & -\sin (\varphi) \sin (\gamma) & \sin (\varphi) \cos (\gamma)
\end{array}\right]\left[\begin{array}{c}
\omega_{i b x}^{b} \\
\omega_{i b y}^{b} \\
\omega_{i b z}^{b}
\end{array}\right]_{b}
$$




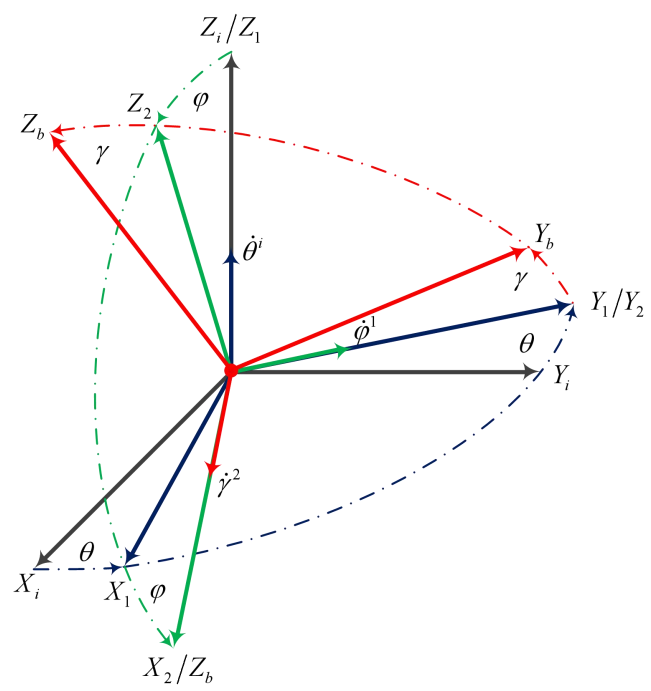

Figure 1. The transformation relation between i-frame and b-frame.

For SINS, there are Euler angle errors $\boldsymbol{\Theta}=\left[\begin{array}{lll}\delta \varphi & \delta \theta & \delta \gamma\end{array}\right]^{T}$ between the head $\varphi^{c}$, pitch $\theta^{c}$, roll $\gamma^{c}$ with respect to computational i-frame $o_{i c} x_{i c} y_{i c} z_{i c}$ and head ${ }^{\varphi}, \operatorname{pitch}^{\theta}$, roll $^{\gamma}$ with respect to i-frame ${ }_{i} x_{i} y_{i} z_{i}$, and can be expressed as: $\boldsymbol{\Theta}=\left[\begin{array}{l}\delta \varphi \\ \delta \theta \\ \delta \gamma\end{array}\right]=\left[\begin{array}{l}\varphi^{c} \\ \theta^{c} \\ \gamma^{c}\end{array}\right]-\left[\begin{array}{l}\varphi \\ \theta \\ \gamma\end{array}\right]$

These Euler angle errors $\boldsymbol{\Theta}=\left[\begin{array}{lll}\delta \varphi & \delta \theta & \delta \gamma\end{array}\right]^{T}$ are different with the misalignment angles $\boldsymbol{\Phi}=\left[\begin{array}{lll}\phi_{x} & \phi_{y} & \phi_{z}\end{array}\right]^{T}$, where $\phi_{j}$ is the misalignment angle between computational i-frame $o_{i c} x_{i c} y_{i c} z_{i c}$ and i-frame $o_{i} x_{i} y_{i} z_{i}(j=x, y, z)$. According to equations (4) and (5), the differential equation of convected Euler angle errors in head, pitch and roll can be obtained. The differential equation of pitch angle error is written by:

$$
\begin{aligned}
& \delta \dot{\theta}=\dot{\theta}^{c}-\dot{\theta}=\frac{1}{\cos \left(\varphi^{c}\right)}\left[\sin \left(\gamma^{c}\right) \omega_{i b y}^{b}+\cos \left(\gamma^{c}\right) \omega_{i b z}^{b}\right]-\frac{1}{\cos \left(\varphi^{c}-\delta \varphi\right)}\left[\sin \left(\gamma^{c}-\delta \gamma\right) \omega_{i b y}^{b}+\cos \left(\gamma^{c}-\delta \gamma\right) \omega_{i b z}^{b}\right] \\
& \approx \frac{1}{\cos \left(\varphi^{c}\right)\left[\cos (\delta \varphi)+\tan \left(\varphi^{c}\right) \delta \varphi\right]}\left\{\left[\begin{array}{l}
{\left[\sin \left(\gamma^{c}\right) \omega_{i b y}^{b}+\cos \left(\gamma^{c}\right) \omega_{i b z}^{b}\right]\left[\cos (\delta \varphi)+\tan \left(\varphi^{c}\right) \delta \varphi\right]-} \\
\left.\left[\sin \left(\gamma^{c}\right)-\cos \left(\gamma^{c}\right) \delta \gamma-\frac{1}{2} \sin \left(\gamma^{c}\right)(\delta \gamma)^{2}\right] \omega_{i b y}^{b}-\left[\cos \left(\gamma^{c}\right)+\sin \left(\gamma^{c}\right) \delta \gamma-\frac{1}{2} \cos \left(\gamma^{c}\right)(\delta \gamma)^{2}\right] \omega_{i b z}^{b}\right\}
\end{array}\right\}\right. \\
& =\frac{1}{\cos \left(\varphi^{c}\right)\left[\cos (\delta \varphi)+\tan \left(\varphi^{c}\right) \delta \varphi\right]}\left\{\begin{array}{l}
{\left[\sin \left(\gamma^{c}\right) \omega_{i b y}^{b}+\cos \left(\gamma^{c}\right) \omega_{i b z}^{b}\right] \tan \left(\varphi^{c}\right) \delta \varphi-\frac{1}{2}\left[\sin \left(\gamma^{c}\right) \omega_{i b y}^{b}+\cos \left(\gamma^{c}\right) \omega_{i b z}^{b}\right](\delta \varphi)^{2}-} \\
{\left[-\cos \left(\gamma^{c}\right) \omega_{i b y}^{b}+\sin \left(\gamma^{c}\right) \omega_{i b z}^{b}\right] \delta \gamma+\frac{1}{2}\left[\sin \left(\gamma^{c}\right) \omega_{i b y}^{b}+\cos \left(\gamma^{c}\right) \omega_{i b z}^{b}\right](\delta \gamma)^{2}}
\end{array}\right\}
\end{aligned}
$$

(6)

Using Taylor series expansion, some trigonometric function can be linearized $\operatorname{as} \sec \left(\varphi^{c}-\delta \varphi\right) \approx \sec \left(\varphi^{c}\right)-\sec \left(\varphi^{c}\right) \tan \left(\varphi^{c}\right) \delta \varphi$, and the differential equation of pitch angle error can be rewritten as:

$$
\delta \dot{\theta}=\left[\sec \left(\varphi^{c}\right)-\sec \left(\varphi^{c}\right) \tan \left(\varphi^{c}\right) \delta \varphi\right]\left\{\begin{array}{l}
{\left[\sin \left(\gamma^{c}\right) \omega_{i b y}^{b}+\cos \left(\gamma^{c}\right) \omega_{i b z}^{b}\right] \tan \left(\varphi^{c}\right) \delta \varphi-\frac{1}{2}\left[\sin \left(\gamma^{c}\right) \omega_{i b y}^{b}+\cos \left(\gamma^{c}\right) \omega_{i b z}^{b}\right](\delta \varphi)^{2}-} \\
{\left[-\cos \left(\gamma^{c}\right) \omega_{i b y}^{b}+\sin \left(\gamma^{c}\right) \omega_{i b z}^{b}\right] \delta \gamma+\frac{1}{2}\left[\sin \left(\gamma^{c}\right) \omega_{i b y}^{b}+\cos \left(\gamma^{c}\right) \omega_{i b z}^{b}\right](\delta \gamma)^{2}}
\end{array}\right\}
$$

Ignoring the term as small as the second order, the differential equation of pitch angle error can be simply written as:

$$
\delta \dot{\theta} \approx \sec \left(\varphi^{c}\right)\left\{\left[\sin \left(\gamma^{c}\right) \omega_{i b y}^{b}+\cos \left(\gamma^{c}\right) \omega_{i b z}^{b}\right] \tan \left(\varphi^{c}\right) \delta \varphi-\left[-\cos \left(\gamma^{c}\right) \omega_{i b y}^{b}+\sin \left(\gamma^{c}\right) \omega_{i b z}^{b}\right] \delta \gamma\right\}
$$

The differential equation of roll angle error can be obtained as: 


$$
\begin{aligned}
\delta \dot{\gamma} & =\dot{\gamma}^{c}-\dot{\gamma} \\
& =\omega_{i b x}^{b}-\sin \left(\gamma^{c}\right) \tan \left(\varphi^{c}\right) \omega_{i b y}^{b}+\cos \left(\gamma^{c}\right) \tan \left(\varphi^{c}\right) \omega_{i b z}^{b}-\left[\omega_{i b x}^{b}-\sin \left(\gamma^{c}-\delta \gamma\right) \tan \left(\varphi^{c}-\delta \varphi\right) \omega_{i b y}^{b}+\cos \left(\gamma^{c}-\delta \gamma\right) \tan \left(\varphi^{c}-\delta \varphi\right) \omega_{i b z}^{b}\right] \\
& =\tan \left(\varphi^{c}\right)\left[-\sin \left(\gamma^{c}\right) \omega_{i b y}^{b}+\cos \left(\gamma^{c}\right) \omega_{i b z}^{b}\right]-\tan \left(\varphi^{c}-\delta \varphi\right)\left[-\sin \left(\gamma^{c}-\delta \gamma\right) \omega_{i b y}^{b}+\cos \left(\gamma^{c}-\delta \gamma\right) \omega_{i b z}^{b}\right]
\end{aligned}
$$

(9)

Based on Taylor series expansion, some trigonometric function can be linearized as $\tan \left(\varphi^{c}-\delta \varphi\right) \approx \tan \left(\varphi^{c}\right)-\sec ^{2}\left(\varphi^{c}\right) \delta \varphi, \quad \sin (\delta \varphi) \approx \delta \varphi, \quad \cos (\delta \gamma) \approx 1-(\delta \gamma)^{2} / 2, \quad \sin (\delta \gamma) \approx \delta \gamma \quad$ and the differential equation of roll angle error can be rewritten as:

$$
\begin{aligned}
\delta \dot{\gamma}= & \tan \left(\varphi^{c}\right)\left\{\left[-\cos \left(\gamma^{c}\right) \omega_{i b y}^{b}-\sin \left(\gamma^{c}\right) \omega_{i b z}^{b}\right] \delta \gamma+\frac{1}{2}\left[-\sin \left(\gamma^{c}\right) \omega_{i b y}^{b}+\cos \left(\gamma^{c}\right) \omega_{i b z}^{b}\right](\delta \gamma)^{2}\right\}+ \\
& \sec ^{2}\left(\varphi^{c}\right) \delta \varphi\left\{\left[-\sin \left(\gamma^{c}\right) \omega_{i b y}^{b}+\cos \left(\gamma^{c}\right) \omega_{i b z}^{b}\right]+\left[\cos \left(\gamma^{c}\right) \omega_{i b y}^{b}+\sin \left(\gamma^{c}\right) \omega_{i b z}^{b}\right] \delta \gamma-\frac{1}{2}\left[\sin \left(\gamma^{c}\right) \omega_{i b y}^{b}-\cos \left(\gamma^{c}\right) \omega_{i b z}^{b}\right](\delta \gamma)^{2}\right\}
\end{aligned}
$$

)

Ignoring the term as small as the second order, the differential equation of roll angle error can be simply written as:

$$
\delta \dot{\gamma}=\tan \left(\varphi^{c}\right)\left[-\cos \left(\gamma^{c}\right) \omega_{i b y}^{b}-\sin \left(\gamma^{c}\right) \omega_{i b z}^{b}\right] \delta \gamma+\sec ^{2}\left(\varphi^{c}\right)\left[-\sin \left(\gamma^{c}\right) \omega_{i b y}^{b}+\cos \left(\gamma^{c}\right) \omega_{i b z}^{b}\right] \delta \varphi
$$

Assuming $\sin (\delta \theta) \approx \delta \theta, \sin (\delta \gamma) \approx \delta \gamma, \cos (\delta \theta) \approx 1-(\delta \theta)^{2} / 2, \cos (\delta \gamma) \approx 1-(\delta \gamma)^{2} / 2$, and the differential equation of head angle error can be written as:

$$
\begin{aligned}
\delta \dot{\varphi} & =\dot{\varphi}^{c}-\dot{\varphi} \\
& =\cos \left(\gamma^{c}\right) \omega_{i b y}^{b}-\sin \left(\gamma^{c}\right) \omega_{i b z}^{b}-\left[\cos \left(\gamma^{c}-\delta \gamma\right) \omega_{i b y}^{b}-\sin \left(\gamma^{c}-\delta \gamma\right) \omega_{i b z}^{b}\right] \\
& =\cos \left(\gamma^{c}\right) \omega_{i b y}^{b}-\sin \left(\gamma^{c}\right) \omega_{i b z}^{b}-\left[\cos \left(\gamma^{c}\right) \cos (\delta \gamma)+\sin \left(\gamma^{c}\right) \sin (\delta \gamma)\right] \omega_{i b y}^{b}+\left[\sin \left(\gamma^{c}\right) \cos (\delta \gamma)-\cos \left(\gamma^{c}\right) \sin (\delta \gamma)\right] \omega_{i b z}^{b} \\
& =[1-\cos (\delta \gamma)]\left[\cos \left(\gamma^{c}\right) \omega_{i b y}^{b}-\sin \left(\gamma^{c}\right) \omega_{i b z}^{b}\right]-\left[\sin \left(\gamma^{c}\right) \omega_{i b y}^{b}+\cos \left(\gamma^{c}\right) \omega_{i b z}^{b}\right] \sin (\delta \gamma)
\end{aligned}
$$

)

Based on Taylor series expansion, $\cos (\delta \gamma) \approx 1-(\delta \gamma)^{2} / 2, \sin (\delta \gamma) \approx \delta \gamma$, and the differential equation of head angle error can be rewritten as:

$\delta \dot{\varphi}=-\left[\sin \left(\gamma^{c}\right) \omega_{i b y}^{b}+\cos \left(\gamma^{c}\right) \omega_{i b z}^{b}\right] \delta \gamma+\left[\cos \left(\gamma^{c}\right) \omega_{i b y}^{b}-\sin \left(\gamma^{c}\right) \omega_{i b z}^{b}\right] \frac{(\delta \gamma)^{2}}{2}$

Ignoring the term as small as the second order, the differential equation of head angle error can be simply written as:

$\delta \dot{\varphi} \approx-\left[\sin \left(\gamma^{c}\right) \omega_{i b y}^{b}+\cos \left(\gamma^{c}\right) \omega_{i b z}^{b}\right] \delta \gamma$

According to equations (8), (11) and (14), the differential equation of convected Euler angle errors can be obtained as:

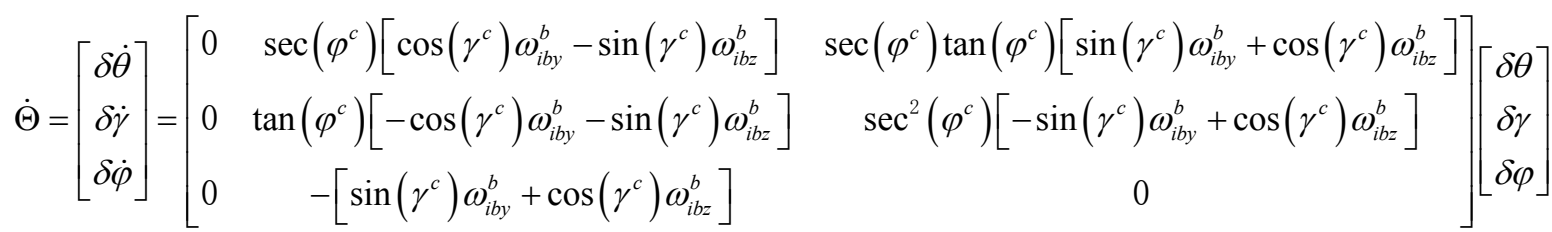

The convected Euler angle error differential equation (15) shown that: (1) The convected Euler angle errors of SINS in i-frame are affected by $\omega_{i b y}^{b}$ and $\omega_{i b z}^{b}$, the angular velocities of b-frame respect to i-frame denoted in b-frame along Y and $\mathrm{Z}$ axes, not $\omega_{i b x}^{b}$ along $\mathrm{X}$ axis. (2) The convected Euler angle errors are affected by head error $\delta \varphi$ and roll error $\delta \gamma$, rather than pitch error $\delta \theta$. (3) The head $\varphi^{c}$ magnitude greatly affects the propagation rules of convected Euler angle errors in pitch and roll but not head.

\section{Attitude determination method based on Kalman filter}

The operation principle of SINS/CNS integrated system is depicted in Figure2. Here, stars observations are 
obtained by star sensors, the Euler angles $\left[\begin{array}{lll}\theta^{M} & \gamma^{M} & \varphi^{M}\end{array}\right]^{T}$ are calculated by CNS attitude matrix ${ }_{i}^{b}$. The gyros output $\left[\begin{array}{lll}\omega_{i b x}^{b} & \omega_{i b y}^{b} & \omega_{i b z}^{b}\end{array}\right]^{T}$ is used to calculate SINS attitude matrix $C_{i}^{b}$, and the Euler angles $\left[\begin{array}{lll}\theta^{S} & \gamma^{S} & \varphi^{S}\end{array}\right]^{T}$ can be obtained. Then, the Euler angle difference $\left.\begin{array}{lll}\delta \theta^{\prime} & \delta \gamma^{\prime} & \delta \varphi^{\prime}\end{array}\right]^{T}$ between SINS and CNS are calculated. The Kalman filter estimates and feeds back the Euler angle errors $\left[\begin{array}{lll}\delta \theta & \delta \gamma & \delta \varphi\end{array}\right]^{T}$ and random drift of gyros $\left[\begin{array}{lll}\varepsilon_{x} & \varepsilon_{y} & \varepsilon_{z}\end{array}\right]^{T}$ in order to compensate SINS. The Euler angle errors $\left[\begin{array}{lll}\delta \theta & \delta \gamma & \delta \varphi\end{array}\right]^{T}$ are considered as the states, and the Euler angle difference $\left.\begin{array}{lll}\delta \theta^{\prime} & \delta \gamma^{\prime} & \delta \varphi^{\prime}\end{array}\right]^{T}$ between SINS and CNS are considered as the measurements in SINS/CNS integrated system. Since the proposed attitude determination method based on convected Euler angle error model avoids the translation between Euler angle errors and misalignment angles, the Euler angle errors $\left.\begin{array}{llll}\delta \theta & \delta \gamma & \delta \varphi\end{array}\right]^{T}$ estimated by the Kalman filter can be compensated directly, which avoids attitude transformation.

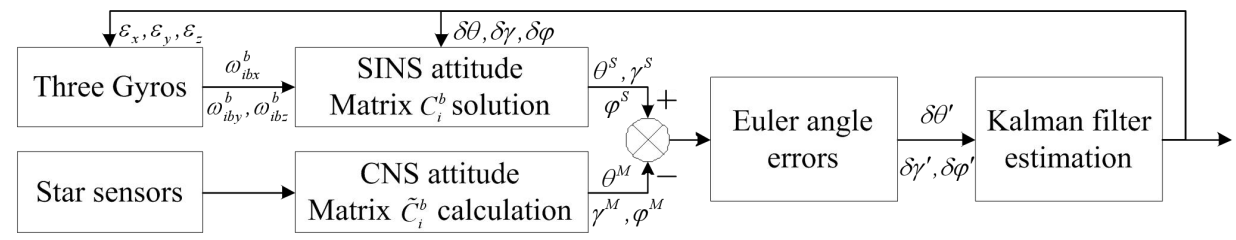

Figure 2. The operation principle of SINS/CNS integrated system.

The state equation and measurement equation are established as follows:

$\left\{\begin{array}{l}\dot{X}=F \cdot X+G \cdot w \\ Z=H X+v\end{array}\right.$

where the state vector $X$ contains 6 states, including Euler angle errors $\left[\begin{array}{lll}\delta \theta & \delta \gamma & \delta \varphi\end{array}\right]^{T}$ and random drift of $\operatorname{gyros}\left[\begin{array}{lll}\varepsilon_{x} & \varepsilon_{y} & \varepsilon_{z}\end{array}\right]^{T}$, and can be given by $X=\left[\begin{array}{llllll}\delta \theta & \delta \gamma & \delta \varphi & \varepsilon_{x} & \varepsilon_{y} & \varepsilon_{z}\end{array}\right]^{T}$. The Euler angle difference $\left[\begin{array}{lll}\delta \theta^{\prime} & \delta \gamma^{\prime} & \delta \varphi^{\prime}\end{array}\right]^{T}$ between SINS and CNS are chosen as the measurement vector $Z$, and can be given by $Z=\left[\begin{array}{lll}\delta \theta^{\prime} & \delta \gamma^{\prime} & \delta \varphi^{\prime}\end{array}\right]^{T}=\left[\begin{array}{lll}\theta^{M}-\theta^{S} & \gamma^{M}-\gamma^{S} & \varphi^{M}-\varphi^{S}\end{array}\right]^{T}, \theta^{M}, \gamma^{M}, \varphi^{M} \quad$ are Euler angles calculated by CNS and $\theta^{S}, \gamma^{S}, \varphi^{S}$ are Euler angles calculated by SINS. $w$ is a zero-mean white-noise vector representing dynamic disturbance noise, and can be given by $w=\left[\begin{array}{llll}w_{\varepsilon_{x}} & w_{\varepsilon_{y}} & w_{\varepsilon_{z}}\end{array}\right]^{T}$. The measurement matrix $H$ can be expressed ${ }_{\text {as }} H=\left[\begin{array}{ll}I_{3 \times 3} & 0_{3 \times 3}\end{array}\right]$. The measurement zero-mean noise $v$ can be expressed as $v=\left[\begin{array}{lll}v_{\delta \theta} & v_{\delta \gamma} & v_{\delta \varphi}\end{array}\right]^{T} . F$ is an $6 \times 6$ matrix, according to equation (15), $F$ can be expressed as follows:

$$
\begin{aligned}
& F=\left[\begin{array}{ll}
A_{3 \times 3} & B_{3 \times 3} \\
0_{3 \times 3} & 0_{3 \times 3}
\end{array}\right] \\
& A_{3 \times 3}=\left[\begin{array}{ccc}
0 & \sec \left(\varphi^{c}\right)\left[\cos \left(\gamma^{c}\right) \omega_{i b y}^{b}-\sin \left(\gamma^{c}\right) \omega_{i b z}^{b}\right] & \sec \left(\varphi^{c}\right) \tan \left(\varphi^{c}\right)\left[\sin \left(\gamma^{c}\right) \omega_{i b y}^{b}+\cos \left(\gamma^{c}\right) \omega_{i b z}^{b}\right] \\
0 & \tan \left(\varphi^{c}\right)\left[-\cos \left(\gamma^{c}\right) \omega_{i b y}^{b}-\sin \left(\gamma^{c}\right) \omega_{i b z}^{b}\right] & \sec ^{2}\left(\varphi^{c}\right)\left[-\sin \left(\gamma^{c}\right) \omega_{i b y}^{b}+\cos \left(\gamma^{c}\right) \omega_{i b z}^{b}\right] \\
0 & -\left[\sin \left(\gamma^{c}\right) \omega_{i b y}^{b}+\cos \left(\gamma^{c}\right) \omega_{i b z}^{b}\right] & 0
\end{array}\right]
\end{aligned}
$$

Since the state vector $X$ include random drift of gyros $\left[\begin{array}{lll}\varepsilon_{x} & \varepsilon_{y} & \varepsilon_{z}\end{array}\right]^{T}$, it is necessary to derive the relation matrix $B_{3 \times 3}$ between Euler angle errors $\left[\begin{array}{lll}\delta \theta & \delta \gamma & \delta \varphi\end{array}\right]^{T}$ and random drift of gyros $\left[\begin{array}{lll}\varepsilon_{x} & \varepsilon_{y} & \varepsilon_{z}\end{array}\right]^{T}$. The gyros actual output $\tilde{\omega}_{i b}^{b}$ includes not only sensing angular velocity $\omega_{i b}^{b}$, but also harmful measurement random drift of gyros $\varepsilon^{b}$. The measurement error can be expressed as:

$\delta \omega_{i b}^{b}=\tilde{\omega}_{i b}^{b}-\omega_{i b}^{b}=\varepsilon^{b}$

According to equations (3), (4) and (17), the differential equation of Euler angle errors induced by angular velocity errors of b-frame respect to i-frame denoted in b-frame, can be written as: 
$\left[\begin{array}{c}\delta \dot{\theta} \\ \delta \dot{\gamma} \\ \delta \dot{\varphi}\end{array}\right] \approx \frac{1}{\cos \left(\varphi^{c}\right)}\left[\begin{array}{ccc}0 & \sin \left(\gamma^{c}\right) & \cos \left(\gamma^{c}\right) \\ \cos \left(\varphi^{c}\right) & -\sin \left(\varphi^{c}\right) \sin \left(\gamma^{c}\right) & \sin \left(\varphi^{c}\right) \cos \left(\gamma^{c}\right) \\ 0 & \cos \left(\varphi^{c}\right) \cos \left(\gamma^{c}\right) & -\cos \left(\varphi^{c}\right) \sin \left(\gamma^{c}\right)\end{array}\right]\left[\begin{array}{c}\varepsilon_{x}^{b} \\ \varepsilon_{y}^{b} \\ \varepsilon_{z}^{b}\end{array}\right]=B_{3 \times 3} \times \varepsilon^{b}$

where $\varphi^{c}, \theta^{c}$ and $\gamma^{c}$ respectively represent head, pitch and roll of b-frame respect to computational i-frame. The relation matrix $B_{3 \times 3}$ can be expressed as:

$$
B_{3 \times 3}=\left[\begin{array}{ccc}
0 & \sec \left(\varphi^{c}\right) \sin \left(\gamma^{c}\right) & \sec \left(\varphi^{c}\right) \cos \left(\gamma^{c}\right) \\
1 & -\tan \left(\varphi^{c}\right) \sin \left(\gamma^{c}\right) & \tan \left(\varphi^{c}\right) \cos \left(\gamma^{c}\right) \\
0 & \cos \left(\gamma^{c}\right) & -\sin \left(\gamma^{c}\right)
\end{array}\right]
$$

According to equation (21), the dynamic noise distribution matrix $G$ can be expressed by $G=\left[\begin{array}{ll}B_{3 \times 3} & 0_{3 \times 3}\end{array}\right]^{T}$.

For linear white Gaussian noise systems, the Kalman filter is the optimal minimum mean square error estimator ${ }^{[18]}$. Kalman filter is designed according to the basic formulae as follows:

$$
\left\{\begin{array}{l}
\hat{X}_{k / k-1}=F_{k, k-1} \hat{X}_{k-1} \\
\hat{X}_{k}=\hat{X}_{k / k-1}+K_{k}\left(Z_{k}-H_{k} \hat{X}_{k / k-1}\right) \\
K_{k}=P_{k / k-1} H_{k}^{T}\left(H_{k} P_{k / k-1} H_{k}^{T}+R_{k}\right)^{-1} \\
P_{k / k-1}=F_{k, k-1} P_{k-1} F_{k, k-1}^{T}+G_{k-1} Q_{k-1} G_{k-1}^{T} \\
P_{k}=\left(I-K_{k} H_{k}\right) P_{k / k-1}\left(I-K_{k} H_{k}\right)^{T}+K_{k} R_{k} K_{k}^{T}
\end{array}\right.
$$

where $\hat{X}_{k / k-1}$ and $\hat{X}_{k}$ are the prior and posterior estimate of the system's state vector respectively, $P_{k / k-1}$ and $P_{k}$ are the corresponding estimate error covariance matrixes. $K_{k}$ is the Kalman gain matrix. $Q_{k}$ and $R_{k}$ are system and measurement noise variance matrices. The estimation accuracy of the Kalman filter depends on a prior knowledge of the system model and the noise statistics.

\section{Simulation experiment for SINS/CNS integrated system}

The SINS/CNS integrated system has features such as strong autonomy and high-attitude precision, and it has become an important development direction for high precision attitude determination of new generation of flying machines including satellite, ballistic missile, and so on, which takes i-frame as the navigation frame. Euler angles, as the direct measurement which can reflect the attitude propagation, are used for navigation, guiding and control. Therefore, the establishment of Euler angle error model is particularly important. In order to validate the proposed attitude determination method based on convected Euler angle error model, a simulation experiment is carried out. The simulation parameters are shown in Table 1 and the simulation results are shown in Figure.3, 4 and 5 .

\begin{tabular}{lc}
\hline Initial term & Initial value \\
\hline Initial latitude and longitude & $110^{\circ} E$ and $40^{\circ} \mathrm{N}$ \\
Initial attitude $\left[\begin{array}{lll}\theta_{0} & \gamma_{0} & \varphi_{0}\end{array}\right]$ & {$\left[\begin{array}{ccc}90^{\circ} & 0^{\circ} & 0^{\circ}\end{array}\right]$} \\
Initial attitude errors $\left[\begin{array}{lll}\delta \theta_{0} & \delta \gamma_{0} & \delta \varphi_{0}\end{array}\right]$ & {$\left[\begin{array}{ccc}0.1^{\circ} & 0.1^{\circ} & 0.15^{\circ}\end{array}\right]$} \\
Constant drift of gyros & $0.01^{\circ} / \mathrm{h}$ \\
Random drift of gyros & $0.01^{\circ} / \mathrm{h}$ \\
Random error of star sensor & $6^{\prime \prime}$ \\
\hline
\end{tabular}

Table 1. The initial parameters. 


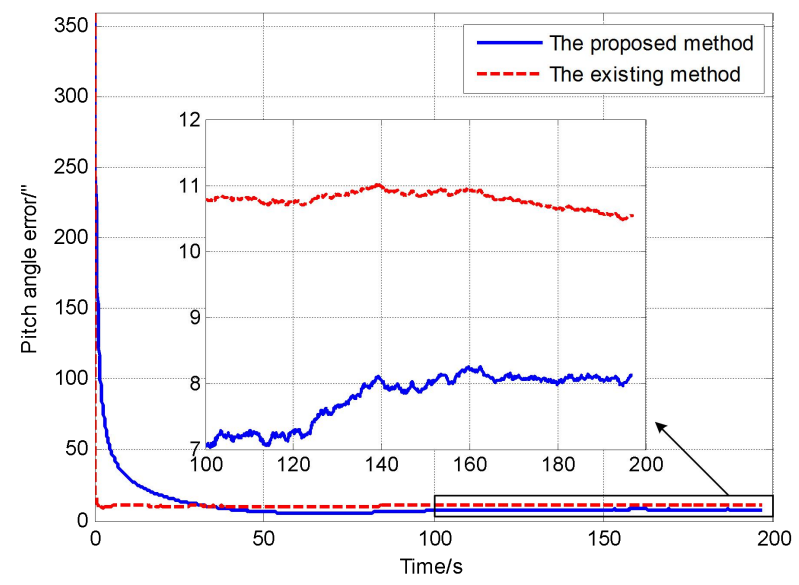

Figure 3. The simulation comparison of pitch angle error.

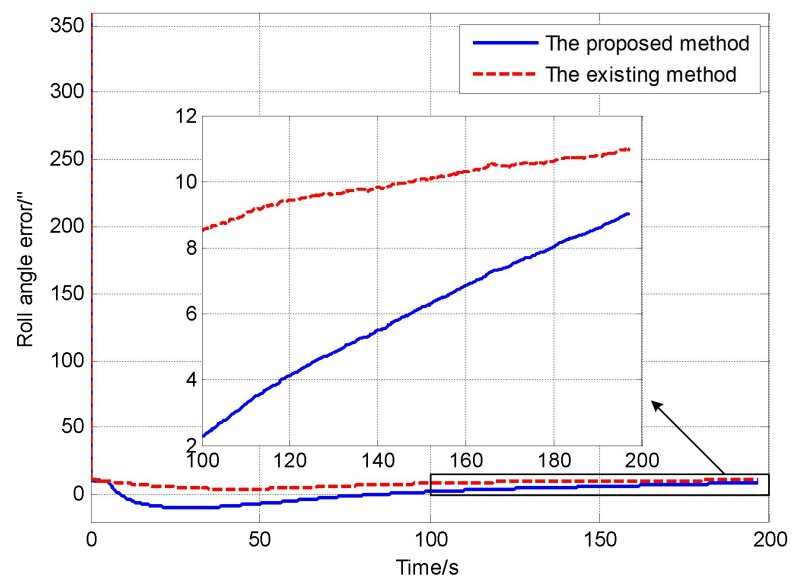

Figure 4. The simulation comparison of roll angle error.

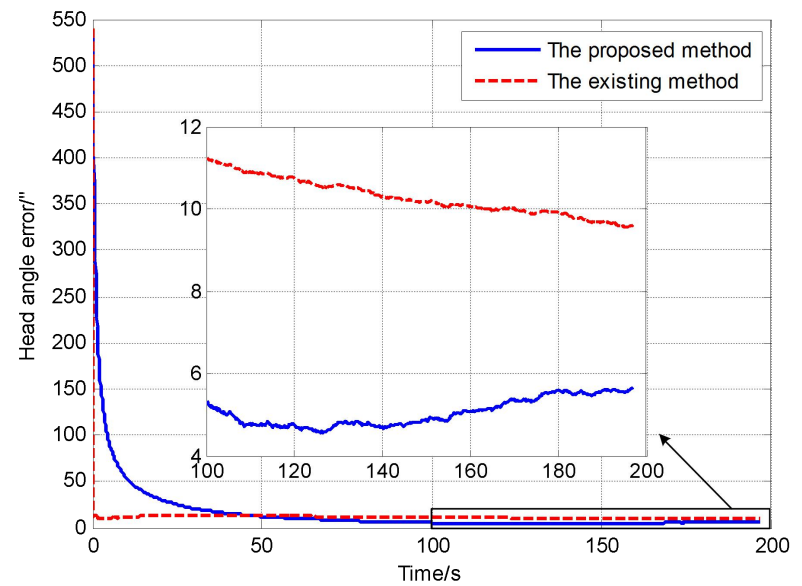

Figure 5. The simulation comparison of head angle error.

\begin{tabular}{llll}
\hline Method & Precision of & Precision of & Precision of \\
& \multicolumn{1}{c}{ pitch error $\delta \theta^{*}\left({ }^{\prime \prime}\right)$} & pitch error $\delta \gamma^{*}\left({ }^{\prime \prime}\right)$ & pitch error $\delta \varphi^{*}\left({ }^{\prime \prime}\right)$ \\
\hline The existing method & 10.65 & 8.76 & 11.08 \\
The proposed method & 7.22 & 5.57 & 7.08 \\
Accuracy improvement & 3.43 & 3.19 & 4.00 \\
\hline
\end{tabular}

Table 2. Comparative analysis of attitude angle errors (RMSE).

As shown in Figure 4, 5, 6 and Table 2, the precision (Root Mean Square Error, RMSE) of pitch, roll and head angle 
errors calculated by the proposed method is superior to $3.43^{\prime \prime}, 3.19^{\prime \prime}, 4.00^{\prime \prime}$ compared to the conventional misalignment angle method. It is obvious that the performance of estimated results is better than the conventional misalignment angle method, and the accuracy improvement of the proposed method comes from the transformation errors caused by conventional misalignment angle method.

\section{Conclusions}

The conventional misalignment angle model can't directly analyze and explain the attitude error propagation, as it is contains attitude transformation errors between misalignment angles and Euler angle errors. The Euler angle errors of SINS are different with misalignment angles of platform inertial navigation system, and a convected Euler angle error item is introduced. The proposed convected Euler angle error model can describe attitude error propagation intuitively, and avoid attitude transformation errors in SINS/CNS integrated system. Simulation experiment show that the precision (RMSE) of head, pith and roll improve $3.19^{\prime \prime}$ at least compared to the conventional misalignment angle method. The attitude determination method based on convected Euler angle error model for SINS/CNS integrated system can realize higher attitude precision. Since the navigation frame is i-frame, the attitude accuracy of other spacecraft can also be improved by this method.

\section{Acknowledgments}

The work described in the current paper was supported by National Natural Science Foundation of China under Grant 61722103, Grant 61721091 and Grant 61571030; in part by the International Cooperation and communication Project under Grant 61661136007. The authors would like to thank all members of Science and Technology on Inertial Laboratory, Fundamental Science on Novel Inertial instrument and Navigation System Technology Laboratory for their useful comments regarding this work.

\section{References}

1. Wang JH, Gao Y. Land vehicle dynamics-aided inertial navigation. IEEE Transaction on Aerospace and Electronic System 2010; 46: 1638-1653.

2. $\mathrm{Li}$ JL, Fang JC, Du M. Error analysis and gyro biases calibration of analytic coarse alignment for airborne POS. IEEE Transactions on Instrumentation and Measurement 2012; 61: 3058-3064.

3. Ning XL, Liu LL. A two-mode INS/CNS navigation method for Lunar Rovers. IEEE Transactions on Instrumentation and Measurement 2014; 63: 2170-2179.

4. Quan W, Li JL, Gong XL, et al. INS/CNS/GNSS integrated navigation technology. New York: Springer Press, 2015.

5. Yang YQ, Zhang CX, Lu JZ. Local observability analysis of star sensor installation errors in a SINS/CNS integration system for near-earth flight vehicles. Sensors $2017 ; 17$.

6. Ali Jamshaid, Fang JC. In-fight alignment of inertial navigation system celestial observation technique. Transactions of Nanjing University of Aeronautics \&amp; Astronautics 2005; 22: 132-138.

7. He Z, Wang XL, Fang JC. An innovative high-precision SINS/CNS deep integrated navigation scheme for the Mars rover. Aerospace Science and Technology 2014; 39: 559-566.

8. Yang YQ, Zhang CX, Lu JZ. Local observability analysis of sar sensor installation errors in a SINS/CNS integration system for near-earth flight vehicles. Sensors 2017; 17: 1-13.

9. Savage PG. Analytical modeling of sensor quantization in strapdown inertial navigation error equations. Journal of Guidance, Control, and Dynamics 2002; 25: 833-842.

10. Hao YL, Gong J, Gao W, et al. Research on the dynamic error of strapdown inertial navigation system. Proceedings of IEEE International Conference on Mechatronics and Automation (ICMA) 2008; 814-819.

11. Chang LB, Li JS, Chen SY. Initial alignment by attitude estimation for strapdown inertial navigation systems. IEEE Transactions on Instrumentation and Measurement 2015; 64: 784-794.

12. Wang R, Xiong Z, Liu JY, et al. Robust inertial-astronomic attitude determination algorithm with adaptive star geometrical error model for HCVs. Journal of Aerospace Engineering 2016; 29: 04015048.

13. Wang MS, Wu WQ, Wang JL, et al. High-order attitude compensation in coning and rotation coexisting environment. IEEE Transaction on Aerospace and Electronic System 2015; 51: 1178-1190.

14. Hamed M, Hadi N, Alireza S. A new approximative method for attitude correction in inertial navigation systems. Proceedings of 2016 7th International Conference on Mechanical and Aerospace Engineering (ICMAE) 2016; 394-399.

15. Ran CY, Cheng XH, Wang L. SINS alignment using velocity matching and simplified Cubature Kalman filter. Applied Mechanics and Materials 2014; 615: 255-258.

16. Janota A, Šimák V, Nemec D, et al. Improving the precision and speed of Euler angles computation from low-cost rotation sensor data. Sensors 2015; 15: 7016-7039.

17. Zhang AH, Bing X, Huo X. Data-driven identification approach for thruster misalignment angles of rigid satellite. 
IET Control Theory and Applications 2015; 9: 1111-1118.

18. Hu GG, Gao SS, Zhong YM, et al. Subic Aleksandar, Modified federated Kalman filter for INS/GNSS/CNS integration. Proceedings of the Institution of Mechanical Engineers Part G-Journal of Aerospace Engineering 2016; 230: $30-44$. 